\title{
Spontaneous polarization in eukaryotic gradient sensing: A mathematical model based on mutual inhibition of frontness and backness pathways
}

\author{
Atul Narang* \\ Department of Chemical Engineering, University of Florida, Gainesville, FL 32611-6005
}

\begin{abstract}
A key problem of eukaryotic cell motility is the signaling mechanism of chemoattractant gradient sensing. Recent experiments have revealed the molecular correlate of gradient sensing: Frontness molecules, such as PI3P and Rac, localize at the front end of the cell, and backness molecules, such as Rho and myosin II, accumulate at the back of the cell. Importantly, this frontness-backness polarization occurs "spontaneously" even if the cells are exposed to uniform chemoattractant profiles. The spontaneous polarization suggests that the gradient sensing machinery undergoes a Turing bifurcation. This has led to several classical activator-inhibitor and activator-substrate models which identify the frontness molecules with the activator. Conspicuously absent from these models is any accounting of the backness molecules. This stands in sharp contrast to experiments which show that the backness pathways inhibit the frontness pathways. Here, we formulate a model based on the mutually inhibitory interaction between the frontness and backness pathways. The model builds upon the mutual inhibition model proposed by Bourne and coworkers (Xu et al, Cell, 114, 201-214, 2003). We show that mutual inhibition alone, without the help of any positive feedback, can trigger spontaneous polarization of the frontness and backness pathways. The spatial distribution of the frontness and backness molecules in response to inhbition and activation of the frontness and backness pathways are consistent with those observed in experiments. Furthermore, depending on the parameter values, the model yields spatial distributions corresponding to chemoattraction (frontness pathways in-phase with the external gradient) and chemorepulsion (frontness pathways out-of-phase with the external gradient). Analysis of the model suggests a mechanism for the chemorepulsion-to-chemoattraction transition observed in neurons.
\end{abstract}

Keywords: Eukaryotic cells, chemotaxis, gradient sensing, directional sensing, spontaneous polarization, Turing instability

\section{Introduction}

When motile cells are exposed to a chemoattractant gradient, they develop a morphological polarity consisting of a distinct front and back [1. The formation of the morphological polarity is driven by the spatial segregation of distinct sets of intracellular molecules to the front and the rear of the cell (see Figure 1ha and refs. 2, 3, 4, 5]). The frontness molecules, which include Cdc42, Rac, PI3P, PI3K, Arp2/3, and F-actin, localize to the front of the cell where they coordinate the extension of an actin-rich extension. The backness molecules, which include Rho, Rho kinase, PTEN, and myosin II, migrate to the rear of the cell where they are thought to activate cell contraction.

In general, the spatial segregation of the frontness/backness molecules and the resultant morphological polarization occurs even if the cells are exposed to a uniform chemoattractant profile (see Figure 1b and [6, 17, 8]). This phenomenon has been called spontaneous polarization to emphasize the fact that the cells polarize despite the absence of a perceptible external cue [9].

The existence of spontaneous polarization is reminiscent of the Turing instability in reaction-diffusion systems [10]. Thus, it has led to several models that view spontaneous polarization, either explicitly or

*Email: narang@che.ufl.edu, URL: http://narang.che.ufl.edu 


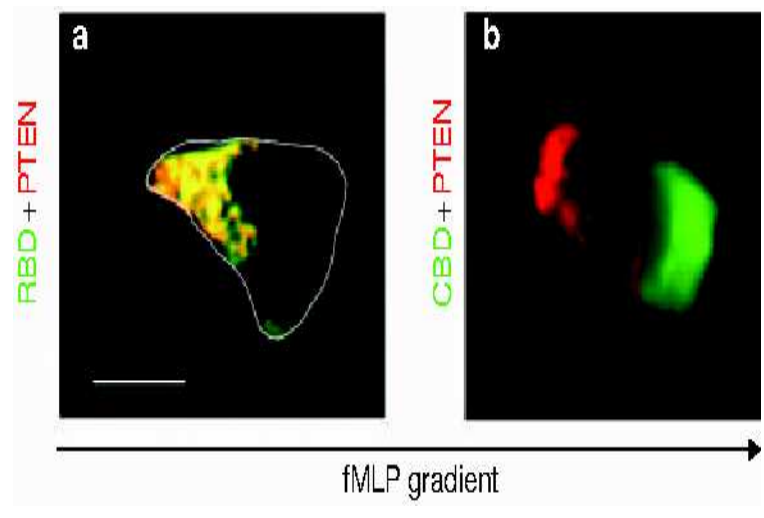

(a)

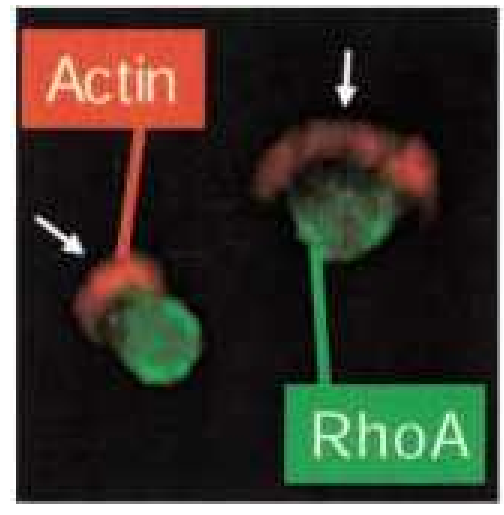

(b)

Figure 1: Spatial segregation of frontness and backness molecules. (a) When neutrophils are exposed to a gradient of fMLP, Rho (represented by its marker, RBD) and PTEN colocalize at the back of the cells, and Cdc42 (represented by its marker, CBD) localizes to the front of the cells (from [5]). (b) The spatial segregation occurs even in the absence of a chemoattractant gradient. When neutrophil-like HL-60 cells are exposed to a uniform concentration (100 nM) of fMLP, actin polymers (shown in red) localize at the front of the cell, and Rho (shown in green) localizes at the back of the cell (from [4]).

implicitly, as the onset of a Turing bifurcation [11, 12, 13, 14, 15]. ${ }^{1}$ According to these models, the cell is in a stable homogeneous steady state in the absence of chemoattractant. However, exposure of the cell to a sufficiently large uniform chemoattractant concentration pushes it past a Turing bifurcation point, where the homogeneous steady state is unstable with respect to certain non-homogeneous perturbations. The inevitable presence of noise is then sufficient to drive the cell towards a nonhomogeneous steady state corresponding to the polarized state of the cell.

As attractive as these models of spontaneous polarization may be, there is a significant gap between the theory and experiments. Indeed, all the models consist of one or more slow-diffusing activators whose synthesis is autocatalytic, and which in consequence, tend to grow and spread across the entire cell. The unconstrained growth and dispersion of the activator is restricted by hypothesizing the existence of a diffusible inhibitor (which is a by-product of activator synthesis and impedes the growth of the activator) or substrate (which is consumed during activator synthesis and stimulates the growth of the activator). The diffusible activator/substrate ensures that the growth of the activator(s) remains confined to a localized region of the cell membrane. This region is identified with the front of the cell, and the rest of the cell membrane, suffering from an activator deficit, is presumed to constitute the back of the cell. The predictions of these models are partially consistent with experiments involving the activation or inhibition of the frontness molecules. Indeed, if PI3K is inhibited, the gradient of the frontness molecules such as PI3P progressively decreases, until at sufficiently high levels of inhibition, there is no gradient at all (Figure 2 2 ). On the other hand, when Rac is overexpressed, high levels of frontness molecules, PI3P and Rac, are found all over the cell membrane (Figure 2 $\mathrm{b}$ ). Both these features are reproduced by the activator-inhibitor class of models [12, 14, 15. However, these models cannot explain the spatial distribution of the backness molecules in response to inhibition of the frontness molecules. Specifically, if the activity of Cdc42 is suppressed, Rho is found not only at the back but also at the front of the cell (Figure 3 a). Likewise, if the Gi proteins are inhibited with pertussis toxin (PTX), a uropod-like structure forms at the up-gradient edge of the cell (Figure $3 \mathrm{~b}$ ). The models also offer no insight into experiments involving activation or inhibition of the backness molecules. For instance,

${ }^{1}$ Alternative models of gradient sensing in which spontaneous polarization plays no role have also been proposed 16 , 17 181920 . For the most part, they are motivated by experimental systems such as PDGF-stimulated fibroblasts 20] and latrunculin-treated Dictyostelium cells [16, which do not display spontaneous polarization. In these models, polarization occurs only in the presence of external gradients. 


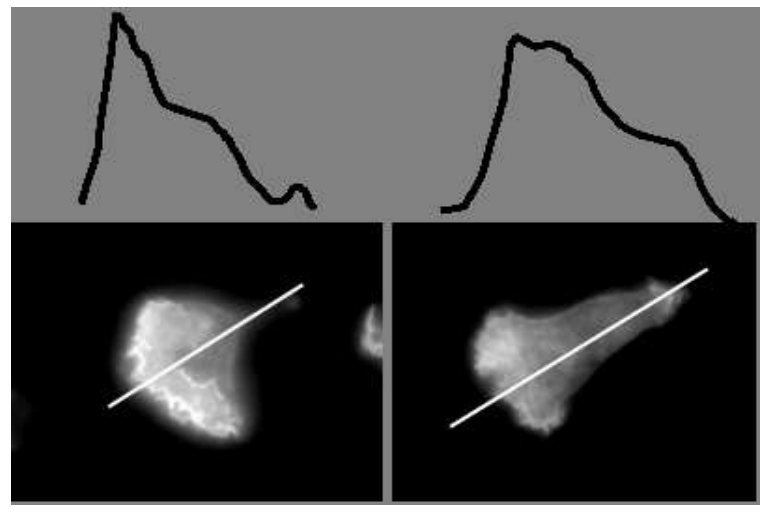

(a)

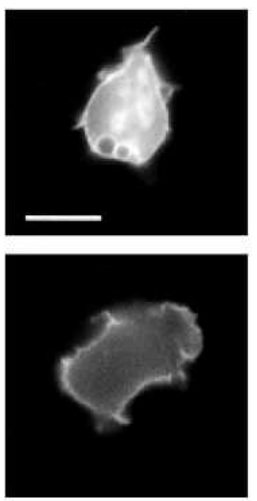

(b)

Figure 2: Spatial redistribution of the frontness pathways in response to inhibition and activation of the frontness pathways. (a) When HL-60 cells are pretreated with intermediate concentrations (100-200 $\mu \mathrm{M})$ of LY294002, an inhibitor of PI3K, before their exposure to fMLP, the gradient of PH-Akt, a marker for PI3P, decreases. The figures on the left and right show the spatial distribution and fluorescence intensity of PH-Akt in control and LY294002-treated cells, respectively. At high concentrations (300 $\mu \mathrm{M})$ of LY294002, PH-Akt does not polarize at all (from [6]). (b) When HL-60 cells transfected with constitutively active Rac are exposed to fMLP, the frontness molecules, PI3P (top) and active Rac (bottom), spread all over the membrane (from [21]).

when Rho is activated, PI3P fails to polarize (Figure 4h). Conversely, when Rho is inhibited, PI3P spreads all over the membrane, and the cell extends a single broad pseudopod or multiple pseudopods (Figure 4b). These results are beyond the scope of the activator-inhibitor and activator-substrate models because the backness molecules are not even acknowledged as legitimate variables - they are implicitly assumed to somehow settle down in regions uninhabited by the frontness molecules. This stands in sharp contrast to the experimental data which shows that backness pathways downregulate the frontness pathways: Inhibition of Rho kinase increases Rac activity $2-3$ fold [4].

One hypothesis regarding the interaction between the frontness and backness pathways is that they inhibit each other [4]. Based on extensive experiments with neutrophils and neutrophil-like HL-60 cells [22, 6, 7, 21, 4], Bourne and coworkers arrived at the kinetic scheme shown in Figure 5 , which they describe as follows

Briefly, the attractant binds to a $\mathrm{G}$ protein-coupled receptor $(\mathrm{R})$, which in turn activates different trimeric $\mathrm{G}$ proteins to generate two divergent, opposing signaling pathways, which promote polarized frontness and backness, respectively. In the frontness pathway, Gi, PI3Ps, and Rac promote de novo formation of actin polymers. One or more positive feedback loops in this first pathway mediate localized increases in sensitivity to attractant: one of these requires polymerized actin, while Rac or Cdc 42 may in addition enhance PI3P accumulation more directly, via an alternative pathway (dotted curved line in Figure 7 [reproduced here as Figure [5] ). Backness signals, generated by G12 and G13, depend on activation of a Rho-dependent pathway that stimulates activation of myosin II, formation of contractile actin-myosin complexes, and myosin-dependent inhibition of Rac- and PI3P-dependent responses. Backness signals inhibit frontness signals, and vice versa (dashed straight lines in Figure 7 ).

They go on to explain spontaneous polarization in terms of this kinetic scheme as follows

The more or less symmetrically distributed actin ruffles and PI3P accumulation seen at early times (e.g., $30 \mathrm{~s}$ ) after application of a uniform stimulus presumably mask a fine-textured mosaic of interspersed backness and frontness signals, some triggering activation of PI3Ks, Rac, and 


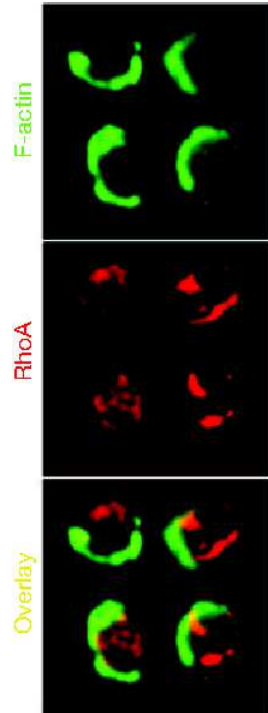

Wt
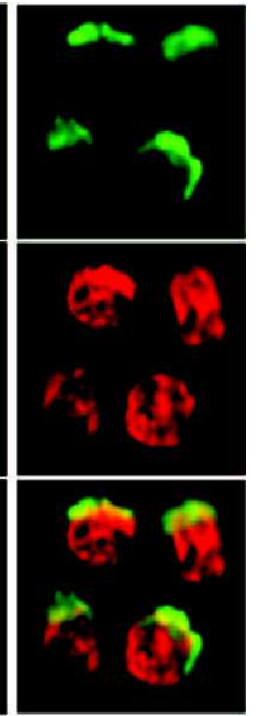

pix
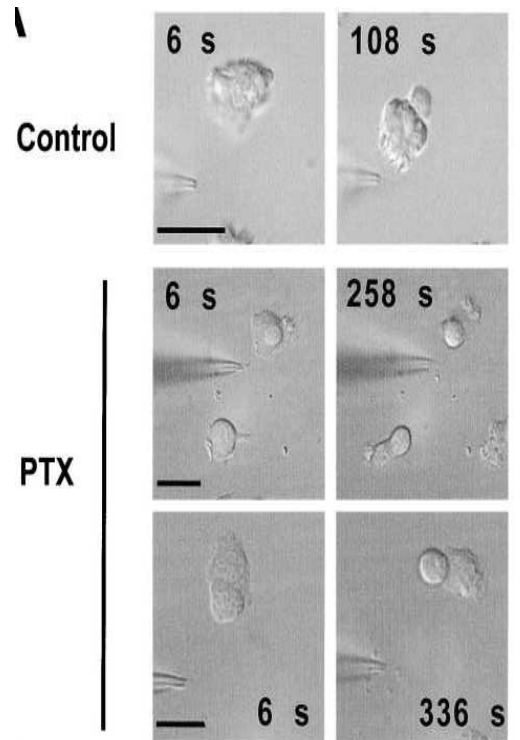

$258 \mathrm{~s}$

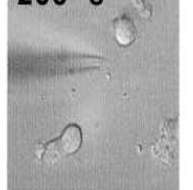

Q.

-

336

(a)

(b)

Figure 3: Spatial redistribution of the backness pathways in response to inhibition of the frontness pathways. (a) When neutrophils devoid of $\alpha$-Pix, an activator of Cdc42, polarize spontaneously, the backness component RhoA is found at the back as well as the front of the cell (along with actin polymers). The panels on the left and right show the distribution of F-actin (green), RhoA (red) and the overlay in wild-type and $\alpha$-Pix-null cells (from [5]). (b) HL-60 cells treated with PTX, a potent inhibitor of Gi proteins, extend a uropod-like structure at the edge exposed to the chemoattractant source (from 4]). In control cells (top panel), a broad "head" is extended toward the fMLP source. In PTX-treated cells (middle and bottom panel), a knob-like structure, similar to the "tail" of control cells, is extended toward the fMLP source. 

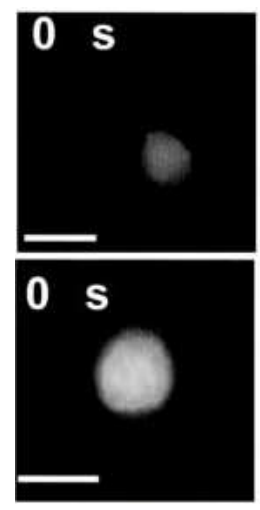
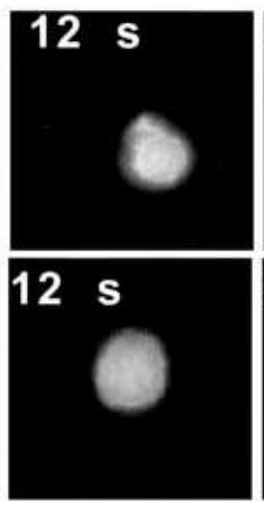

(a)
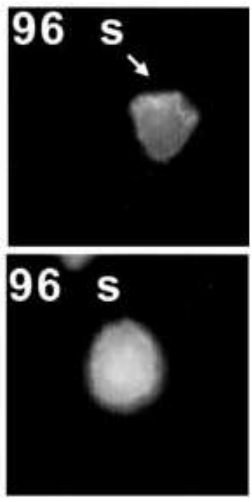
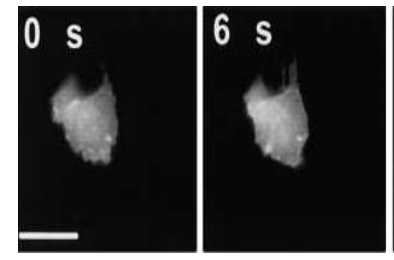
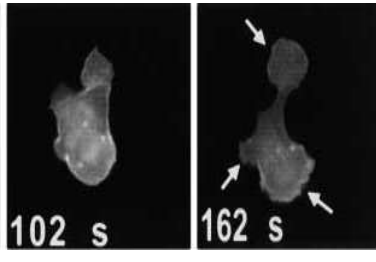

(b)

Figure 4: Spatial redistribution of the frontness and backness pathways in response to inhibition and activation of the backness pathways (from [4]). (a) When HL-60 cells transfected with constitutively active Rho are exposed to fMLP, there is no polarization of PI3P. The top and lower panel show the evolution of the spatial distribution of PI3P in wild-type and transfected cells. (b) When HL-60 cells transfected with dominant negative Rho are exposed to a uniform concentration of fMLP, PH-Akt, a marker for PI3P, spreads all over the membrane, and multiple pseudopods develop.

actin polymerization, others promoting activation of Rho and myosin. Localized mechanical incompatibility of the two cytoskeletal responses, combined with the ability of each to damp signals that promote the other (dashed inhibitor lines in Figure 7), then gradually drive them to separate into distinct domains of the membrane.

The goal of this work is to formulate a mathematical model of spontaneous polarization based on the foregoing mechanism, namely, mutual inhibition of the frontness and backness pathways.

It turns out that the Bourne model imposes two requirements that are incompatible with Turing instabilities in a two-component system consisting of frontness and backness pathways. Specifically, the mutual inhibition between the frontness and backness pathways must be sufficiently strong to ensure that the homogeneous steady state is unstable in the presence of diffusion, and yet weak enough to guarantee its stability in the absence of diffusion. These two requirements cannot be satisfied simultaneously in a two-component system, a point that will be discussed in Section 3

Thus, we are led to consider the modification of the Bourne model shown in Figure $5 \mathrm{~b}$. It contains two variables, $U_{2}, U_{3}$, representing the frontness and backness pathways, respectively, whose activation is driven by receptor ligation, and which inhibit each other in a concentration-dependent manner. It is assumed furthermore that the mutual inhibition between $U_{2}$ and $U_{3}$ is so strong that they are mutually incompatible (in a sense that will be made mathematically precise below). We refer to these two variables as activators. The model differs from the Bourne picture inasmuch as it assumes the existence of a diffusible inhibitor, denoted $U_{1}$, whose synthesis is promoted by both activators, but which, in turn, inhibits both activators. The inhibitor serves to stabilize the coexistence of the otherwise incompatible activators: Transient increases in activator concentrations at localized "hotspots" are efficiently suppressed by the concominant increase in the concentration of the inhibitor. However, the mobility of the inhibitor imposes constraints upon its stabilizing effect because it tends to diffuse away from the "hotspot." This is not an issue at low chemoattractant concentrations, for under these conditions, the activator concentrations, and hence, their mutual incompatibility, is so small that despite the high diffusibility of the inhibitor, it successfully damps fluctuations of the activator levels. However, at high chemoattractant concentrations, the activator concentrations and their mutual incompatibility are so large that the diffusible inhibitor fails to suppress the two activators sufficiently. The mutual incompatibility of the two activators now overcomes the mollifying effect of the inhibitor, and the activators segregate spatially into separate domains. 


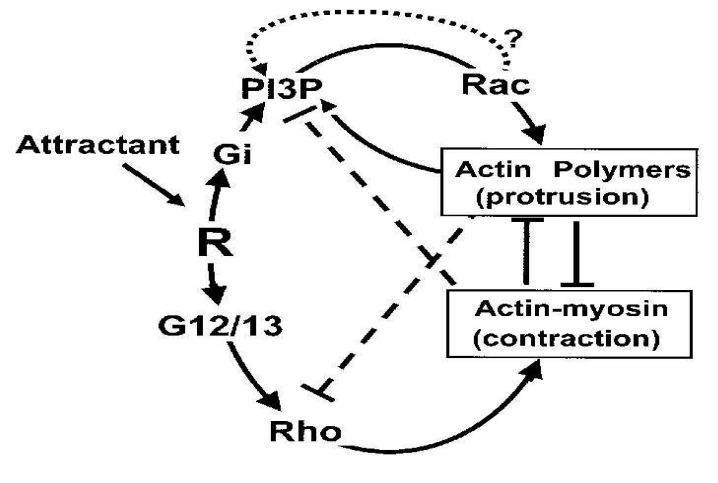

(a)

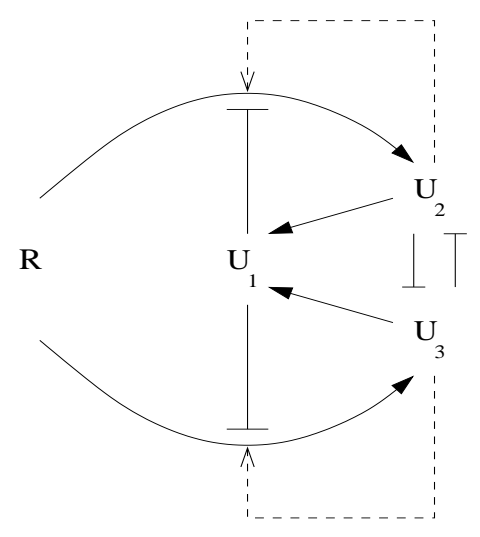

(b)

Figure 5: Model schemes. (a) Model proposed by Bourne and co-workers for spontaneous polarization of neutrophils and HL-60 cells (from [4]). The chemoattractant binds to G-protein-coupled receptors, R. The Gi-coupled receptors activate the frontness pathway which includes PI3P, Rac, and actin polymers. The G12/13-coupled receptors activate the backness pathway which includes Rho and myosin. The frontness pathway is subject to positive feedback from actin polymers (full line from actin polymers to PI3P) and possibly Rac (dashed line from Rac to PI3P). The frontness and backness pathways inhibit each other (dashed lines with a bar at the end). (b) The model scheme considered in this paper. Receptor activation stimulates the synthesis of frontness and backness subsystems, denoted $U_{2}$ and $U_{3}$, respectively. The frontness and backness subsystems stimulate their own synthesis (dashed lines), but inhibit each other both directly (line from $U_{2}$ to $U_{3}$, and vice versa), and indirectly through production of the inhibitor, $U_{1}$. 
The mathematical model in this paper quantifies the above physical argument. We show that the model yields spontaneous polarization at sufficiently high active receptor levels. Moreover, if the frontness or backness pathways are inhibited or activated, they redistribute spatially in a manner consistent with the experiments described above. Finally, we show the model displays steady states corresponding to both chemoattraction and chemorepulsion. Interest in this phenomenon is motivated by the fact that when neurons are exposed to activators of the cGMP pathway, chemorepulsion turns into chemorepulsion 23. Based on the analysis of the model, we suggest that such transitions can be triggered by altering the balance of power in the mutually inhibitory interactions between the frontness and backness pathways.

The mechanism of spontaneous polarization in this model is distinct from that in activator-inhibitor or activator-substrate models. Unlike these models, the spatial segregation of the two activators is driven entirely by their mutual inhibition - positive feedback plays no role. In reality, both positive feedback and mutual inhibition cooperate to produce symmetry-breaking (Figure 5 ). The contribution of this work is to highlight the distinct role of mutual inhibition, a feature that emphasized in the experimental literature [24, 4], but absent from previous activator-inhibitor and activator-substrate models.

The paper is organized as follows. In Section 2] we define the model and simulate the experiments described above. In Section 3, we elaborate on the physics underlying the model. Finally, we summarize the conclusions.

\section{Results}

\subsection{The model}

We assume that

1. Both activators promote the synthesis of the inhibitor, which in turn, degrades by a first-order process. Thus, the net rate of synthesis of $U_{1}$ is

$$
-R_{1} u_{1}+A_{12} u_{2}+A_{13} u_{3} .
$$

2. The synthesis of $U_{2}$ is receptor-mediated. It is autocatalytic at low concentrations and self-limiting at high concentrations, i.e.,

$$
R_{2} r u_{2}-A_{22} u_{2}^{2}
$$

where $r$ denotes the receptor activity, and the second term represents a self-limiting process that prevents synthesis rate from increasing beyond bounds.

3. The synthesis of $U_{2}$ is inhibited in a concentration-dependent manner by the common inhibitor, $U_{1}$, and the other activator, $U_{3}$. Assuming that these interactions follow bimolecular kinetics, the net rate of synthesis of $U_{2}$ is

$$
R_{2} r u_{2}-A_{22} u_{2}^{2}-A_{21} u_{1} u_{2}-A_{23} u_{2} u_{3} .
$$

4. The synthesis of $U_{3}$ follows kinetics similar to those of $U_{2}$, i.e., its net rate of synthesis is

$$
R_{3} r u_{3}-A_{33} u_{3}^{2}-A_{31} u_{1} u_{3}-A_{32} u_{2} u_{3}
$$

Given these assumptions, we arrive at the equations

$$
\begin{aligned}
& \frac{\partial u_{1}}{\partial T}=D_{1} \frac{\partial u_{1}}{\partial X^{2}}-R_{1} u_{1}+A_{12} u_{2}+A_{13} u_{3} \\
& \frac{\partial u_{2}}{\partial T}=D_{2} \frac{\partial u_{2}}{\partial X^{2}}+\left(R_{2} r-A_{21} u_{1}-A_{23} u_{3}-A_{22} u_{2}\right) u_{2} \\
& \frac{\partial u_{3}}{\partial T}=D_{3} \frac{\partial u_{3}}{\partial X^{2}}+\left(R_{3} r-A_{31} u_{1}-A_{32} u_{2}-A_{33} u_{3}\right) u_{3}
\end{aligned}
$$

where $X$ denotes the spatial coordinate, $T$ denotes time, and $u_{1}, u_{2}, u_{3}$ denote the concentrations of $U_{1}, U_{2}, U_{3}$, respectively. We assume the Neumann boundary conditions

$$
\frac{\partial u_{i}}{\partial X}=0 \text { at } x=0, L
$$




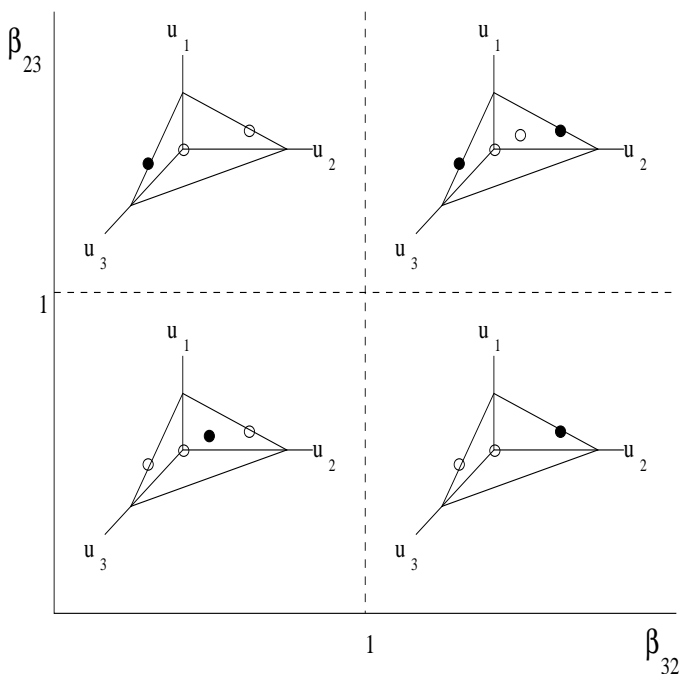

(a)

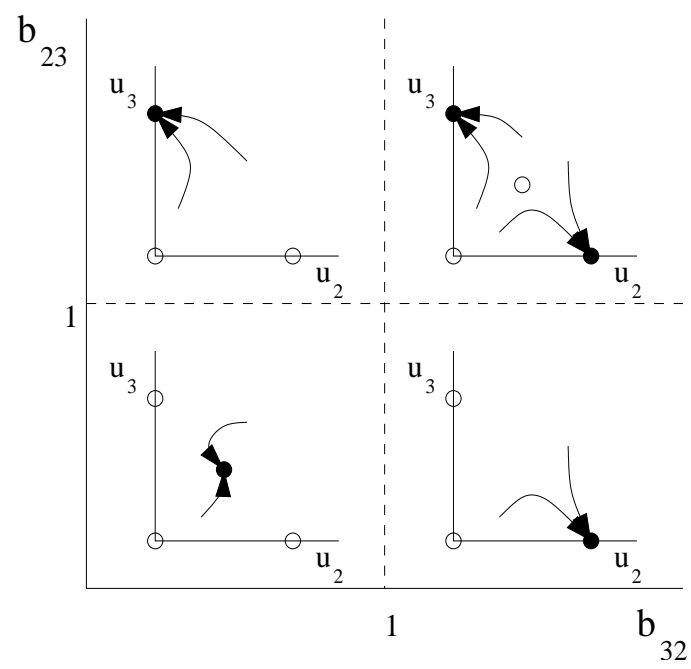

(b)

Figure 6: The bifurcation diagram for the homogeneous steady states (a) In the presence of the inhibitor and (b) in the absence of the inhibitor. The stable and unstable steady states are represented by full and open circles, respectively.

since they imply no interaction with the environment, which is consistent with our desire to study spontaneous (autonomous) polarization.

It is convenient to rescale the equations. If we define

$$
\begin{gathered}
t=a_{11} T, x=\frac{X}{L}, \quad d_{i}=\frac{D_{i} / L^{2}}{R_{1}}, \\
a_{i j}=\frac{A_{i j}}{R_{i}}, \rho_{2}=\frac{R_{2}}{R_{1}}, \quad \rho_{3}=\frac{R_{3}}{R_{1}},
\end{gathered}
$$

we obtain the scaled equations

$$
\begin{aligned}
& \frac{\partial u_{1}}{\partial t}=d_{1} \frac{\partial u_{1}}{\partial x^{2}}-u_{1}+a_{12} u_{2}+a_{13} u_{3} \\
& \frac{\partial u_{2}}{\partial t}=d_{2} \frac{\partial u_{2}}{\partial x^{2}}+\rho_{2}\left(r-a_{21} u_{1}-a_{22} u_{2}-a_{23} u_{3}\right) u_{2} \\
& \frac{\partial u_{3}}{\partial t}=d_{3} \frac{\partial u_{3}}{\partial x^{2}}+\rho_{3}\left(r-a_{31} u_{1}-a_{32} u_{2}-a_{33} u_{3}\right) u_{3}
\end{aligned}
$$

and boundary conditions

$$
\frac{\partial u_{i}}{\partial x}=0 \text { at } x=0,1 .
$$

Our goal is to study the variation of the steady states as a function of the receptor activity, $r$. We shall show, in particular, that the model has a homogeneous steady state at which $u_{2}$ and $u_{3}$ coexist, but it becomes Turing unstable at a sufficiently large $r$. The non-homogeneous steady state emerging from the Turing bifurcation is such that $u_{2}$ and $u_{3}$ are spatially segregated.

\subsection{The homogeneous steady states}

A Turing instability occurs when a non-homogeneous steady state bifurcates from a homogeneous steady state that is stable in the absence of diffusion. It is therefore useful to characterize the conditions for stability of the homogeneous steady states in the absence of diffusion. 
The model has three types of homogeneous steady states: the trivial steady state, $u_{1}=u_{2}=u_{3}=0$, denoted $E_{000}$; the semitrivial steady states, $u_{1}, u_{2}>0, u_{3}=0$, and $u_{1}, u_{3}>, u_{2}=0$, denoted $E_{110}$ and $E_{101}$, respectively; and the nontrivial steady state, $u_{1}, u_{2}, u_{3}>0$, denoted $E_{111}$. The key results, depicted graphically in Figure 6 , are as follows (see Appendix $\$$ for details):

1. The trivial steady state, $E_{000}$, exists for all $r>0$, and is always unstable.

2. The semi-trivial steady state, $E_{110}$, which lies on the $u_{1} u_{2}$-plane, exists for all $r>0$. It is stable if and only if

$$
\beta_{32} \equiv \frac{\alpha_{32}}{\alpha_{22}}>1 .
$$

Here, $\alpha_{22} \equiv a_{22}+a_{21} a_{12}$ is a measure of the extent to which $U_{2}$ inhibits itself both directly $\left(a_{22}\right)$ and indirectly through production of $U_{1}\left(a_{21} a_{12}\right)$. Likewise, $\alpha_{32} \equiv a_{32}+a_{31} a_{12}$ is a measure of the extent to which $U_{2}$ inhibits $U_{3}$ directly $\left(a_{32}\right)$ and indirectly through production of $U_{1}\left(a_{31} a_{12}\right)$. The foregoing stability condition then says that $E_{110}$ is stable if and only if $U_{2}$ inhibits $U_{3}$ more than it inhibits itself.

3. The semi-trivial steady state, $E_{101}$, which lies on the $u_{1} u_{3}$-plane, exists for all $r>0$. It is stable if and only if

$$
\beta_{23} \equiv \frac{\alpha_{23}}{\alpha_{33}}>1,
$$

where $\alpha_{23} \equiv a_{23}+a_{21} a_{13}, \alpha_{33} \equiv a_{33}+a_{31} a_{13}$ are measures of the extent to which $U_{3}$ inhibits $U_{2}$ and itself, respectively, and $\beta_{23}$ is measure of the cross-inhibition relative to the self-inhibition.

4. The coexistence steady state, $E_{111}$, exists if and only if

$$
\beta_{23}, \beta_{32}<1 \text { or } \beta_{23}, \beta_{32}>1 \text {. }
$$

It is stable only if the mutual inhibition of $U_{2}$ and $U_{3}$ is sufficiently weak, i.e., $\beta_{23} \beta_{32}<1$.

Figure 6 is reminiscent of the bifurcation diagram for the Lotka-Volterra model [25]. This is not surprising because $U_{2}$ and $U_{3}$ obey Lotka-Volterra dynamics in the absence of the inhibitor. Indeed, the equations obtained by letting $u_{1}=0$ in equations (5, 6) are identical to the Lotka-Volterra model for two competing species. It follows that the dynamics of $U_{2}$ and $U_{3}$ in the absence of the inhibitor are described by a bifurcation diagram very similar to Figure 6 a, the only difference being that $\beta_{23}$ and $\beta_{32}$ must now be replaced by $b_{23} \equiv a_{23} / a_{22}, b_{32} \equiv a_{32} / a_{33}$ (see Figure $6 \mathrm{~b}$ ). We shall appeal to this fact below.

\subsection{Turing instability of homogeneous steady states}

Since $E_{000}$ is always unstable, it can never undergo a Turing bifurcation. However, the semi-trivial and non-trivial steady states are stable for all sufficiently small $r>0$. The question then arises whether these steady states can undergo a Turing bifurcation. It is shown in Appendix B that

1. The semi-trivial steady states cannot undergo a Turing bifurcation.

2. The nontrivial steady state, $E_{111}$, can undergo a Turing bifurcation, but this is so only if

$$
b_{23} b_{32}>1,
$$

i.e., the mutual inhibition between $U_{2}$ and $U_{3}$ must be sufficiently strong - so strong, in particular, that they cannot coexist in the absence of the inhibitor (see Figure 6 b).

Both conclusions are a consequence of the following fact which will be discussed in Section 3 In this model, the only destabilizing mechanism driving the Turing instability is mutual inhibition of $U_{2}$ and $U_{3}$, which requires the existence of both activators. Thus, the semi-trivial steady states fail to undergo a Turing bifurcation because one of the two activators is absent at such steady states. The non-trivial steady state, which is characterized by positive concentrations of $U_{2}$ and $U_{3}$, allows for a Turing instability, but only if their mutual inhibition is sufficiently strong. 
Although the mutual inhibition must be sufficiently strong to ensure that $E_{111}$ undergoes a Turing instability, intuition suggests that it cannot be too strong, lest the two activators become incompatible even in the presence of the inhibitor. This is indeed the case. To see this, observe that (7) can be satisfied in three different ways (see Figure 6 b):

1. $b_{23}, b_{32}>1$, i.e., the mutual inhibition between $U_{2}$ and $U_{3}$ is so strong that they display bistable dynamics in the absence of the inhibitor.

2. $b_{23}<1, b_{32}>1$, i.e., in the absence of the inhibitor, $U_{2}$ inhibits $U_{3}$ much more than $U_{3}$ inhibits $U_{2}$, so that $U_{2}$ ultimately prevails over $U_{3}$.

3. $b_{23}>1, b_{32}<1$, i.e., in the absence of the inhibitor, $U_{3}$ inhibits $U_{2}$ much more than $U_{2}$ inhibits $U_{3}$, so that $U_{3}$ ultimately prevails over $U_{2}$.

It turns out that in the first case, when both $U_{2}$ and $U_{3}$ inhibit each other strongly, the necessary condition (17) is satisfied. Yet, the Turing instability cannot be realized because a stable coexistence steady state fails to exist even in the presence of the inhibitor. This follows from the fact that $E_{111}$ exists and is stable only if the mutual inhibition is sufficiently weak in the presence of the inhibitor, i.e.,

$$
\beta_{23}=\frac{a_{23}+a_{21} a_{13}}{a_{33}+a_{31} a_{13}}<1, \beta_{32}=\frac{a_{32}+a_{31} a_{12}}{a_{22}+a_{21} a_{12}}<1
$$

which can be recast in the form

$$
\frac{a_{22}}{a_{12}}\left(b_{32}-1\right)<a_{21}-a_{31}<\frac{a_{33}}{a_{13}}\left(1-b_{23}\right) .
$$

Clearly, (8) cannot be satisfied if $b_{23}, b_{32}>1$. Under these conditions, the mutual inhibition of $U_{2}$ and $U_{3}$ is so strong that they cannot coexist stably even in the presence of the inhibitor.

We conclude that $E_{111}$ exists and bifurcates via a Turing instability only if the interaction between $U_{2}$ and $U_{3}$ in the absence of the inhibitor is such that only one of them prevails ultimately. Furthermore

1. If $U_{2}$ prevails over $U_{3}$ in the absence of the inhibitor $\left(b_{23}<1, b_{32}>1\right)$, then a Turing instability obtains only if $b_{23} b_{32}>1$ and

$$
0<\frac{a_{22}}{a_{12}}\left(b_{32}-1\right)<a_{21}-a_{31}<\frac{a_{33}}{a_{13}}\left(1-b_{23}\right) .
$$

2. Conversely, if $U_{3}$ prevails over $U_{2}$ in the absence of the inhibitor $\left(b_{23}>1, b_{32}<1\right)$, then a Turing instability obtains only if $b_{23} b_{32}>1$ and

$$
\frac{a_{22}}{a_{12}}\left(b_{32}-1\right)<a_{21}-a_{31}<\frac{a_{33}}{a_{13}}\left(1-b_{23}\right)<0 .
$$

These conditions have a simple physical interpretation. Consider, for instance, the condition (9). It says that if $U_{2}$ prevails over $U_{3}$ in the absence of the inhibitor, a Turing instability obtains only if (a) $a_{21}-a_{31}>0$, i.e., $U_{1}$ inhibits $U_{2}$ more than it inhibits $U_{3}$. The stronger inhibition of $U_{2}$ by $U_{1}$ is necessary in order to compensate for its intrinsic superiority over $U_{3}$. (b) The magnitude of this difference must be neither too small nor too large to prevent under- or over-compensation that would preclude the coexistence of $U_{2}$ and $U_{3}$ even in the presence of the inhibitor.

It is shown in Appendix B that the conditions (9) or (10) are not only necessary but almost sufficient for $E_{111}$ to undergo a Turing instability. It suffices to impose the additional condition that $d_{1}$ be sufficiently larger than $d_{2}$ and $d_{3}$.

\subsection{Simulation of experiments}

To simulate the data shown in Figure 1b, wherein the frontness and backness components segregate spontaneously in response to a uniform chemoattractant profile, we chose parameter values satisfying (9), shown in the column labeled " $U_{2}$ wins" of Table 1. Linear stability analysis shows that given these parameter values, the coexistence steady state, $E_{111}$, undergoes a Turing instability at $r \approx 0.6$ and wavenumber $k \approx 1$ 
Table 1: Parameter values for the simulations. The diffusion coefficient of the inhibitor, $D_{1}$, is assumed to much larger than the diffusion coefficients, $D_{2}, D_{3}$, of the frontness and backness pathways. The rate constants, $R_{i}, A_{i j}$, were chosen in order to satisfy the conditions (9) or (10). These two cases are shown in the columns labeled column labeled " $U_{2}$ wins" and " $U_{3}$ wins", respectively.

\begin{tabular}{|c|c|c|c|c|c|}
\hline Parameter & $U_{2}$ wins & $U_{3}$ wins & Parameter & $U_{2}$ wins & $U_{3}$ wins \\
\hline \hline$D_{1}$ & 3 & 3 & $A_{13}$ & $1 / 3$ & 4 \\
\hline$D_{2}$ & 0.001 & 0.001 & $A_{21}$ & 3 & 1 \\
\hline$D_{3}$ & 0.005 & 0.005 & $A_{22}$ & 1 & 1 \\
\hline$R_{1}$ & 1 & 1 & $A_{23}$ & 1 & 2 \\
\hline$R_{2}$ & 2 & 1 & $A_{31}$ & 1 & 3 \\
\hline$R_{3}$ & 1 & 2 & $A_{32}$ & 2 & 1 \\
\hline$A_{12}$ & 4 & $1 / 2$ & $A_{33}$ & 1 & 1 \\
\hline
\end{tabular}

(Figure [1]). Computations with the continuation software package CONTENT [26] confirm the existence of this instability (Figure $7 \mathrm{a}$ ). The homogeneous steady state is stable for $0<r \lesssim 0.6$, and Turing unstable therafter. The concentration profiles of the non-homogeneous steady state created at the Turing bifurcation point are consistent with the data. Figure $7 \mathrm{~b}$ shows that the non-homogeneous steady state at $r=1$ is such that $U_{2}$ and $U_{1}$ are in phase, and $U_{2}$ and $U_{3}$ are out of phase. The latter is consistent with the data in Figure 1 $1 \mathrm{~b}$. We refer to this steady state as the chemoattraction steady state for the following reason. To a first degree of approximation, the steady state obtained in the presence of a receptor gradient, $r(x)=1+\epsilon \cos (\pi x), 0<\epsilon \ll 1$, is identical to the steady state shown in Figure $7 \mathrm{~b}$. Thus, the profile of the frontness pathways is in phase with the distribution of the active receptors, which is characteristic of chemoattraction.

Figures 2 and 3 show the redistribution of the frontness and backness pathways in response to inhibition and activation of the frontness pathways. To simulate these experiments, we computed the variation of the chemoattraction steady state at $r=1$ with respect to $R_{2}$. Figure 8 a shows that as $R_{2}$ decreases, so does $u_{2}^{\max }$ until it becomes zero, i.e., the chemoattraction steady state ceases to exist, and is replaced by the homogeneous steady state, $E_{101}$, in which $u_{2}=0$ throughout the spatial domain. The variation of the concentration profile for the frontness pathway is consistent with the data shown in Figure 2 Figures $8 \mathrm{~b}$ shows that as $R_{2}$ decreases, so does the gradient of the frontness molecules until it vanishes at $R_{2} \approx 1.5$, which is in qualitative agreement with the data shown in Figure 2 . Conversely, at sufficiently large values of $R_{2}$, the non-homogeneous steady state is replaced by the homogeneous steady state, $E_{110}$, characterized by high levels of the frontness molecules uniformly distributed throughout the spatial domain (see Figure 2b). The variation of the concentration profile for the backness pathway is also consistent with the data. Figures 8 : shows that as $R_{2}$ decreases, the backness pathway progressively advances into the pre-existing front until it occupies not only the back but also the front of the cell. This is consistent with the data shown in Figure 3 a.

Simulations in which $r$ is held at 1.0 and $R_{3}$ is progressively changed give analogous results (simulations not shown). As $R_{3}$ increases, the frontness pathways occupy a progressively smaller portion of the cell until there is no frontness throughout the cell. The simulations did not yield multiple peaks corresponding to the multiple pseudopods shown in Figure $4 \mathrm{~b}$. Numerical calculations of the critical wavenumber, $k_{0}$, show that it increases as $R_{3}$ decreases, which is the correct trend. However, the steady state ceases to exist before $k_{0}$ can exceed 2.

To investigate the existence of steady states corresponding to chemorepulsion, we chose parameter values satisfying (10) so that $U_{3}$ prevails over $U_{2}$ in the absence of the inhibitor (column labeled " $U_{3}$ wins" of Table (1). Here, the coexistence steady state, $E_{111}$, undergoes a Turing bifurcation at $r \approx 0.65$ and $k=1$ (Figures [1]). The non-homogeneous steady state that emerges from this bifurcation is such that $U_{2}$ and $U_{3}$ are once again out of phase, ${ }^{2}$ but it is $U_{3}$, instead of $U_{2}$, that is in phase with $U_{1}$ (Figure 9b). This non-homogeneous steady state represents the chemorepulsion steady state, since it approximates the steady

\footnotetext{
${ }^{2}$ It is shown in Appendix B that regardless of the parameter values, $U_{2}$ and $U_{3}$ will always be out-of-phase in the nonhomogeneous steady state bifurcating from $E_{111}$.
} 


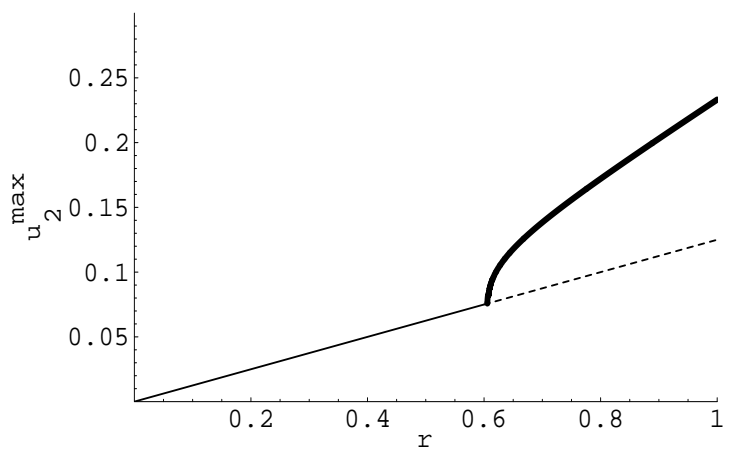

(a)

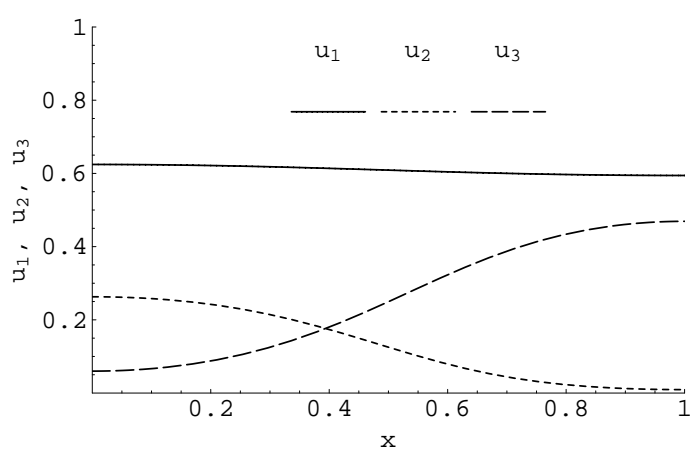

(b)

Figure 7: Symmetry-breaking of the non-trivial steady state when the parameters are chosen such that $U_{2}$ wins in the absence of the inhibitor. (a) The bifurcation diagram depicting the variation of $u_{2}^{\max } \equiv$ $\max _{0 \leq x \leq 1} u_{2}(x)$ as a function of the active receptor concentration, $r$. The thin line represents the homogeneous non-trivial steady state, $E_{111}$; the dashed portion of this line indicates that $E_{111}$ is Turing unstable. The thick line represents the non-homogeneous steady state emerging from the Turing bifurcation at $r \approx 0.6$. (b) The profiles of $u_{1}, u_{2}$, and $u_{3}$ at the non-homogeneous steady state corresponding to $r=1$. The steady state was attained by perturbing the homogeneous steady state at $r=1$, i.e., by integrating equations (4) 6) with initial conditions, $u_{1}(0, x)=\tilde{u}_{1}, u_{i}(0, x)=\tilde{u}_{i}[1+0.01 \cos (\pi x)], i=2,3$, where $\tilde{u}_{i}=1,2,3$ denotes the non-trivial homogeneous steady state at $r=1$.

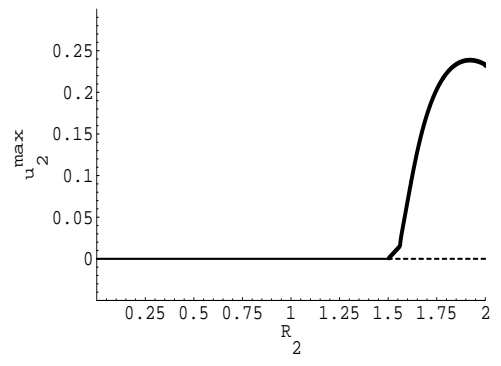

(a)

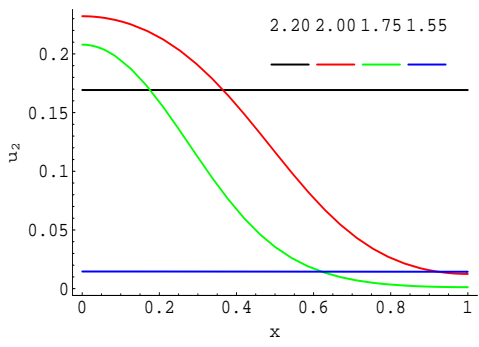

(b)

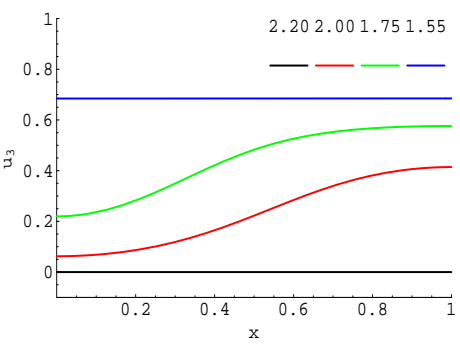

(c)

Figure 8: Variation of the chemoattraction steady state with respect to $R_{2}$ at $r=1$. (a) The bifurcation diagram showing the variation of $u_{2}^{\max }$ as a function of $R_{2}$. The thick line represents the non-homogeneous steady state. The thin line represents the homogeneous semi-trivial steady state, $E_{101}$. As $R_{2}$ decreases, so does $u_{2}^{\max }$ until it intersects the branch, $u_{2}^{\max }=0$, which corresponds the homogeneous semi-trivial steady state, $E_{101}$. (b) As $R_{2}$ decreases, the concentration profile of $U_{2}$ decreases in magnitude and occupies a progressively smaller proportion of the cell until $u_{2}$ is identically zero. (c) As $R_{2}$ decreases, the concentration profile of $U_{3}$ increases in magnitude and advances into the pre-existing front until $u_{3}$ acquires the uniform profile of $E_{101}$. 


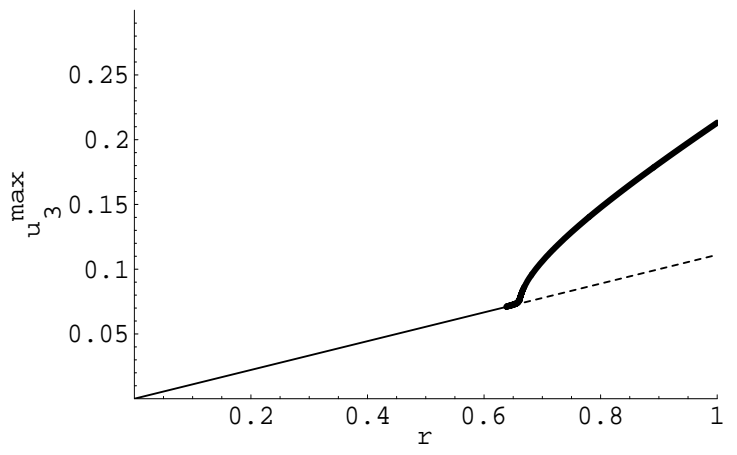

(a)

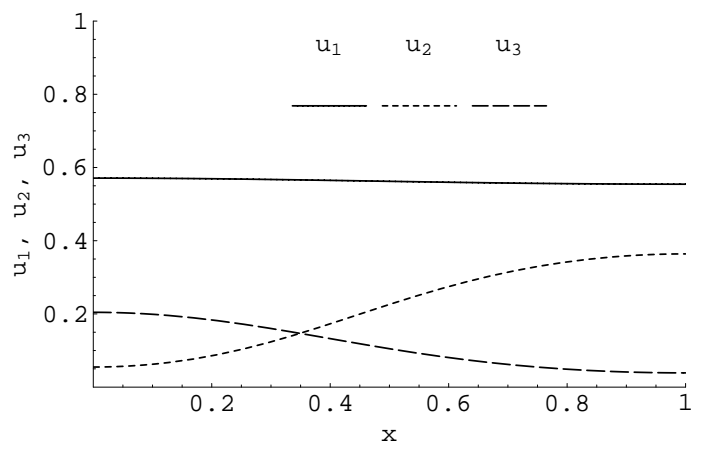

(b)

Figure 9: Symmetry-breaking of the non-trivial steady state when the parameters are chosen such that $U_{3}$ wins in the absence of the inhibitor. (a) The bifurcation diagram depicting the variation of $u_{3}^{\max } \equiv$ $\max _{0 \leq x \leq 1} u_{3}(x)$ as a function of the active receptor concentration, $r$. The thin line represents the homogeneous non-trivial steady state, $E_{111}$; the dashed portion of this line indicates that $E_{111}$ is Turing unstable. The thick line represents the non-homogeneous steady state emerging from the Turing bifurcation at $r \approx 0.65$. (b) The profiles of $u_{1}, u_{2}$, and $u_{3}$ at the non-homogeneous steady state attained by perturbing the homogeneous steady state at $r=1$.

state that would be obtained in the presence of a receptor gradient, $r(x)=1+\epsilon \cos (\pi x), 0<\epsilon \ll 1$, and the profile of the frontness pathways are, in this case, out of phase with the active receptor distribution. Given the symmetry of the equations, it is not surprising that the model admits a chemorepulsion steady state. For, if choose the parameters corresponding to the column " $U_{2}$ wins" in Table 1 but interchange $u_{2}$ and $u_{3}$, the chemoattraction steady state in Figure $7 \mathrm{~b}$ turns into a chemorepulsion steady state. However, the analysis of the model suggests a mechanism for the transition. Specifically, the conditions (910) imply that transitions from chemorepulsion to chemattraction, such as those observed in neurons [23], can be triggered by pharmacological agents that selectively inhibit the backness pathway so that the frontness pathway becomes intrinsically superior in the absence of the inhibitor.

\section{Discussion}

We have shown that a model based on mutual inhibition of the frontness and backness pathways can yield spontaneous polarization involving spatial segregation of the two pathways. The frontness and backness pathways are mutually incompatible in the absence of the inhibitor (eq. 7), but coexist in the presence of the inhibitor because it suppresses the growth, and hence, the mutual incompatibility, of the frontness and backness pathways. Since the inhibitor is diffusible, it can suppress the nominal incompatibility manifested at low receptor levels. However, it fails to achieve this goal at high receptor levels, resulting in spatial segregation of the frontness and backness pathways (see Figures 1] and 7).

Since the model takes due account of the backness pathways, we could explore the effect of frontness inhibition or activation on the backness pathways, and the effect of backness modification on the frontness and backness pathways. The simulations in Section 2 yielded results that are in qualitative agreement with the data shown in Figures 14 These results are beyond the scope of activator-inhibitor or activator-substrate models of gradient sensing since they do not account for the backness pathways.

The particular manner in which the two pathways segregate depends on their behavior in the absence of the tempering influence of the inhibitor. If the frontness pathways dominate over the backness pathways, their spatial segregation is consistent with chemoattraction. Conversely, if the backness pathways dominate, 
the emergent spatial pattern corresponds to chemorepulsion. The model therefore suggests that transitions between chemoattraction and chemorepulsion can be provoked by agents that shift the balance of power between the frontness and backness pathways.

The model proposed here is the simplest possible model of spontaneous polarization driven by mutual inhibition. By this, we mean the following

1. A model containing a smaller number of variables will not yield spontaneous polarization.

2. The model is simpler than any other model in which the symmetry-breaking is driven entirely by mutual inhibition.

In what follows, we justify these conclusions, since they are required to explain certain points raised above.

To see that a model containing 2 variables will not yield spontaneous polarization driven by mutual inhibition, let $u=\left[u_{1}, u_{2}\right]^{t}$ be a homogeneous steady state of a two-component reaction-diffusion system

$$
\frac{\partial u_{i}}{\partial t}=D_{i} \frac{\partial u_{i}}{\partial x^{2}}+f_{i}\left(u_{1}, u_{2}\right), i=1,2
$$

and let $J(u)$ denote the Jacobian at $u$. Then, $u$ can undergo a Turing instability only if [27]

$$
J_{11}+J_{22}<0, J_{11} J_{22}-J_{12} J_{21}>0, D_{1} J_{22}+D_{2} J_{11}>0 .
$$

The first and third conditions imply that $J_{11} J_{22}<0$, i.e., one of the components, say $U_{1}$, must inhibit its own synthesis $\left(J_{11}<0\right)$, and the other, say $U_{2}$, must activate its own synthesis $\left(J_{22}>0\right)$. Now, if the two components inhibit each other, then $J_{12}, J_{21}<0$, so that $J_{12} J_{21}>0$ and $J_{11} J_{22}-J_{12} J_{21}<0$, which violates the second condition for Turing stability. Thus, a two-component model in which each component inhibits the other component cannot yield spontaneous polarization. Indeed, such a model can yield a Turing instability only if $J_{12} J_{21}<0$, i.e., the interaction between $U_{1}$ and $U_{2}$ is asymmetric. If $U_{1}$ inhibits $U_{2}$, then $U_{2}$ must activate $U_{1}$; this corresponds to the activator-inhibitor model; conversely, if $U_{1}$ activates $U_{2}$, then $U_{2}$ must inhibit $U_{1}$, which corresponds to the activator-substrate model. No other cross-interactions are admissible. It follows that if spontaneous polarization is to be driven by mutual inhibition, there must be at least three components. Thus, we were led to modify the Bourne model by postulating the existence of an additional component, namely, a diffusible inhibitor.

To show that the model is simpler than any other model in which symmetry-breaking is driven entirely by mutual inhibition, we appeal to the fact that in a general reaction-diffusion system, a homogeneous steady state cannot become Turing instable unless it contains an unstable subsytem [28. ${ }^{3}$ The three-component model considered here has three 1-component subsystems, $\left\{U_{1}, U_{2}, U_{3}\right\}$, and three 2-component subsystems, $\left\{U_{1} U_{2}, U_{2} U_{3}, U_{1} U_{3}\right\}$. It turns out that in the neighborhood of the non-trivial steady state, $E_{111}$, all three 1-component subsystems are stable, i.e. $J_{11}, J_{22}, J_{33}<0$ (see 13). In other words, the self-interactions of the individual components are stabilizing. Notably, $U_{2}$ and $U_{3}$ are stable subsystems even though their synthesis was assumed to be autocatalytic. This is because the kinetics of their synthesis are such that the feedback is positive only when $u_{2}$ and $u_{3}$ are small. However, in the neighborhood of $E_{111}, u_{2}$ and $u_{3}$ are so large that the self-limiting effect dominates, and the feedback is, in fact, negative. Now, since the 1-component subsystems are stable, $E_{111}$ can be Turing unstable only if at least one of the 2-component subsystems is unstable, i.e., the cross-interaction of at least one 2-component system is destabilizing. Such destabilizing cross-interactions can occur only if the interaction between the 2 components is mutually inhibitory or synergistic, i.e.,

$$
J_{12} J_{21}>0 \text { or } J_{13} J_{31}>0 \text { or } J_{23} J_{32}>0 \text {. }
$$

The first two conditions cannot be satisfied since the interaction between $U_{1}$ and each of the two activators, $U_{2}$ and $U_{3}$ is neither mutually inhibitory nor mutually synergistic $-U_{1}$ inhibits $U_{2}$ and $U_{3}$, but $U_{2}$ and $U_{3}$

\footnotetext{
${ }^{3}$ A subsystem refers to any proper subset of the model variables. For example, the two-component model discussed above has two subsystems, $\left\{U_{1}, U_{2}\right\}$. A subsystem is said to be unstable if it becomes unstable as soon as the remaining components of the system are somehow rendered constant. In other words, without the stabilizing effects of the other variables, the subsystem diverges from its values at the homogeneous steady state. In the 2-component model, the activator is an unstable subsystem: If the inhibitor or substrate level is somehow held constant, the activator diverges from the homogeneous steady state due to positive feedback $\left(J_{22}>0\right)$. As shown above, the existence of such an unstable subsystem is necessary for the full system to undergo a Turing instability.
} 
activate $U_{1}$. Thus, $\left\{U_{1}, U_{2}\right\}$ and $\left\{U_{1}, U_{3}\right\}$ are stable subsystems, and $\left\{U_{2}, U_{3}\right\}$ is the only unstable subsystem in the model. Consequently, the spatial segregation of the frontness and backness pathways is driven entirely by mutual inhibition between $U_{2}$ and $U_{3}$ - positive feedback plays no role since $J_{11}, J_{22}, J_{33}<0$.

It should be noted that reciprocal distribution of the frontness and backness pathways (specifically, PI3K and PTEN) is a central feature of the local-excitation-global-inhibition model proposed by Iglesias and coworkers 19. However, the mechanism for spatial segregation of PI3K and PTEN is such that polarization occurs only in the presence of a chemoattractant gradient. Indeed, according to this model

1. Exposure to chemoattractant generates binding sites for PI3K and PTEN at the membrane.

2. The rate of generation of binding sites for PI3K and PTEN is proportional to $r^{2}$ and $r$, respectively, where $r$ denotes the active receptor concentration.

3. The concentration of PI3P is determined by the relative concentrations of membrane-bound PI3K and PTEN - the higher the PI3K:PTEN ratio, the larger the concentration of PI3P.

It follows from (2) that when a cell is exposed to a gradient, the leading edge develops a high PI3K:PTEN ratio, while the trailing edge has a high PTEN:PI3K ratio. Then, (3) implies that the PI3P concentration at the leading edge is higher than that at the trailing edge. Importantly, the polarized distribution of PI3P arises only in the presence of a gradient. For, if $r$ is constant, the PI3K:PTEN ratio, and hence, the concentration of PI3P, is constant all over the cell membrane. Thus, the local-excitation-global-inhibition model cannot capture the spontaneous polarization observed in the absence of gradients.

Although our model captures spontaneous polarization driven by mutual inhibition of frontness and backness pathways, it is missing two important features of the experimental data. First, it is known that both negative and positive feedback operate in chemoattractant-mediated polarization (see Figure 5h and refs. [24, 4]). Although we assumed the existence of positive feedback in the model, it played no role in the spontaneous polarization since $J_{22}, J_{33}<0$ in the neighborhood of $E_{111}$. Second, the model does not account for adapation, the process that enables the cell to ultimately return to the homogeneous steady state even in the presence of chemoattractant [29. A modified model taking due account of positive feedback and long-term adaptation is currently under investigation.

Two of the model variables $\left(U_{2}, U_{3}\right)$ correspond to the frontness and backness pathways of the Bourne scheme. However, the model hypothesizes the existence of an additional variable, namely, a diffusible inhibitor required to suppress the mutual incompatibility of the frontness and backness pathways. It is, by no means, necessary that this diffusible component be an inhibitor. It could just as well be a diffusible substrate that is required for the synthesis of the two activators, but is consumed in process of activator synthesis. This would reverse the signs of $J_{12}, J_{13}, J_{21}, J_{31}$, but the key inequalities in the above arguments, $J_{13} J_{31}, J_{12} J_{21}<0$, would be preserved. A diffusible inhibitor was assumed only because there is some evidence of their existence.

1. Luo and coworkers have shown that cytosolic inositol phosphates, whose levels surge immediately after chemoattractant stimulation, inhibit the frontness pathways in both Dictyostelium 30 and neutrophillike HL-60 cells [31].

2. Nimnual et al have shown that Rac-mediated production of reactive oxygen species downregulates the backness component, Rho 32 .

It remains to be seen if these diffusible inhibitors play a critical role in the maintenance of polarity, as required by the diffusible inhibitor of the proposed model.

\section{Conclusions}

We have formulated a model that provides a mathematical realization of the Bourne scheme for spontaneous polarization by mutual inhibition of frontness and backness pathways [4. The model predicts several experimentally observed features that are outside the scope of prevailing models of gradient sensing. 
1. Mutual inhibition of the frontness and backness pathways plays a critical role in generating the spontaneous polarization.

2. If the frontness pathway is suppressed, the backness pathways occupy a progressively larger proportion of the cell. Conversely, if the backness pathway is suppressed, the frontness pathways invade and occupy the previous back of the cell.

3. Depending on the parameter values, the model yields steady states corresponding to both chemoattraction and chemorepulsion. Analysis of the model suggests that chemorepulsion-to-chemoattraction transitions observed in neurons can be triggered by agents that inhibit the backness pathways more than the frontness pathways.

The model provides a useful starting point for formulating models that account for the positive and negative feedback effects, both of which have been shown to play a role in gradient sensing.

\section{Acknowledgments}

I would like to thank Prof. D. A. Lauffenburger for helpful comments on the manuscript.

\section{References}

[1] Douglas A. Lauffenburger and Alan F. Horwitz. Cell migration: A physically integrated molecular process. Cell, 84:359-369, 1996.

[2] S. Funamoto, R. Meili, S. Lee, L. Parry, and R. A. Firtel. Spatial and temporal regulation of 3phosphoinositides by PI 3-Kinase and PTEN mediates chemotaxis. Cell, 109:611-623, 2002.

[3] M. Iijima and P. N. Devreotes. Tumor suppressor PTEN mediates sensing of chemoattractant gradients. Cell, 109:599-610, 2002.

[4] J. Xu, F. Wang, A. Van Keymeulen, P. Herzmark, A. Straight, K. Kelly, Y. Takuwa, N. Sugimoto, T. Mitchison, and H. R. Bourne. Divergent signals and cytoskeletal assemblies regulate self-organizing polarity in neutrophils. Cell, 114:201-214, 2003.

[5] Zhong Li, Xuemei Dong, Xiemei Dong, Zhenglong Wang, Wenzhong Liu, Ning Deng, Yu Ding, Liuya Tang, Tim Hla, Rong Zeng, Lin Li, and Dianqing Wu. Regulation of PTEN by Rho small GTPases. Nat Cell Biol, 7(4):399-404, Apr 2005.

[6] F. Wang, P. Herzmark, O. D. Weiner, S. Srinivasan, G. Servant, and H. R. Bourne. Lipid products of PI(3)Ks maintain persistent cell polarity and directed motility in neutrophils. Nat. Cell Biol., 4:513-518, 2002.

[7] O. D. Weiner, P. O. Neilsen, G. D. Prestwich, M. W. Kirschner, L. C. Cantley, and H. R. Bourne. A PtdInsP(3)- and Rho GTPase-mediated positive feedback loop regulates neutrophil polarity. Nat. Cell Biol., 4:509-513, 2002.

[8] M. Postma, J. Roelofs, J. Goedhart, T. W. J. Gadella, A. J. W. J. Visser, and P. J. M. Van Haastert. Uniform cAMP stimulation of Dictyostelium cells induces localized patches of signal transduction and pseudopodia. Mol. Biol. Cell, 14:5019-5027, 2003.

[9] R. Wedlich-Soldner, S. Altschuler, L. Wu, and R. Li. Spontaneous cell polarization through actomyosinbased delivery of the Cdc42 GTPase. Science, 299:1231-1235, 2003.

[10] A. M. Turing. The chemical basis of morphogenesis. Phil. Trans. Roy. Soc. Lond. B, 237:37-72, 1952.

[11] Hans Meinhardt. Orientation of chemotactic cells and growth cones: Models and mechanisms. J. Cell Sc., 112:2867-2874, 1999. 
[12] A. Narang, K. K. Subramanian, and D. A. Lauffenburger. A mathematical model for chemoattractant gradient sensing based on receptor-regulated membrane phospholipid signaling dynamics. Ann Biomed Eng, 29(8):677-91, Aug 2001.

[13] M. Postma and P. J. M. Van Haasert. A diffusion-translocation model for gradient sensing by chemotactic cells. Biophys. J., 81:1314-1323, 2001.

[14] Ivan V Maly, H Steven Wiley, and Douglas A Lauffenburger. Self-organization of polarized cell signaling via autocrine circuits: computational model analysis. Biophys J, 86(1 Pt 1):10-22, Jan 2004.

[15] K. K. Subramanian and Atul Narang. A mechanistic model for eukaryotic gradient sensing: spontaneous and induced phosphoinositide polarization. J Theor Biol, 231(1):49-67, Nov 2004.

[16] Andre Levchenko and A. Pablo Iglesias. Models of Eukaryotic Gradient Sensing: Application to Chemotaxis of Amoebae and Neutrophils. Biophys. J., 82:50-63, 2002.

[17] W-J. Rappel, P. J. Thomas, H. Levine, and W. F. Loomis. Establishing direction during chemotaxis in eucaryotic cells. Biophys. J., 83:1361-1367, 2002.

[18] J. Krishnan and Pablo A. Iglesias. Analysis of the signal transduction properties of a module of spatial sensing in eukaryotic chemotaxis. Bull. Math. Biol., 65:95-128, 2003.

[19] Lan Ma, Chris Janetopoulos, Liu Yang, Peter N Devreotes, and Pablo A Iglesias. Two complementary, local excitation, global inhibition mechanisms acting in parallel can explain the chemoattractant-induced regulation of PI(3,4,5)P3 response in dictyostelium cells. Biophys J, 87(6):3764-74, Dec 2004.

[20] Ian C. Schneider and Jason M. Haugh. Spatial analysis of 3' phosphoinositide signaling in living fibroblasts: Ii. parameter estimates for individual cells from experiments. Biophys. J., 86:599-608, 2004.

[21] Supriya Srinivasan, Fei Wang, Suzana Glavas, Alexander Ott, Fred Hofmann, Klaus Aktories, Daniel Kalman, and H. R. Bourne. Rac and cdc42 play distinct roles in regulating pi $(3,4,5) \mathrm{p} 3$ and polarity during neutrophil chemotaxis. J Cell Biol, 160(3):1-11, 2003.

[22] Guy Servant, Orion D. Weiner, Enid R. Neptune, John W. Sedat, and Henry R. Bourne. Dynamics of a chemoattractant receptor in living neutrophils during chemotaxis. Mol. Biol. Cell, 10:1163-1178, 1999.

[23] HJ Song, GL Ming, and MM Poo. cAMP-induced switching in turning direction of nerve growth cones. Nature, 388(6639):275-9, Jul 1997.

[24] Ruedi Meili and Richard A Firtel. Two poles and a compass. Cell, 114(2):153-6, Jul 2003.

[25] J. D. Murray. Mathematical Biology. Biomathematics Texts. Springer-Verlag, New York, 1989.

[26] Yu. A. Kuznetsov. CONTENT - Integrated environment for analysis of dynamical systems, 20 March 1998.

[27] Lee A. Segel and Julius L. Jackson. Dissipative structure: An explanation and an ecological example. J. Theor. Biol., 37:545-559, 1972.

[28] R. A. Satnoianu, M. Menzinger, and P. K. Maini. Turing instabilities in general systems. J Math Biol, 41(6):493-512, Dec 2000.

[29] Carole A. Parent, B. J. Blacklock, W. M. Froehlich, D. B. Murphy, and Peter N. Devreotes. G protein signaling events are activated at the leading edge of cells. Cell, 95:81-91, 1998.

[30] H. R. Luo, Y. E. Huang, J. C. Chen, A. Saiardi, M. Iijima, K. Ye, Y. Huang, E. Nagata, P. Devreotes, and S. H. Snyder. Inositol pyrophosphates mediate chemotaxis in Dictyostelium via pleckstrin homology domain-PIP 3 interactions. Cell, 114:559-572, 2003.

[31] Hongbo Luo. Ins $(1,3,4,5) \mathrm{p} 4$ as a negative regulator of neutrophil chemotaxis. Poster presented at the Gordon Conference on "Gradient Sensing and Directed Cell Migration" held Feb 20-25, 2005.

[32] Anjaruwee S Nimnual, Laura J Taylor, and Dafna Bar-Sagi. Redox-dependent downregulation of Rho by Rac. Nat Cell Biol, 5(3):236-41, Mar 2003. 


\section{A Existence/stability of homogeneous steady states}

The homogeneous steady states of (4-6) satisfy the equations

$$
\begin{aligned}
-u_{1}+a_{12} u_{2}+a_{13} u_{3} & =0 \\
\left(r-a_{21} u_{1}-a_{22} u_{2}-a_{23} u_{3}\right) u_{2} & =0 \\
\left(r-a_{31} u_{1}-a_{32} u_{2}-a_{33} u_{3}\right) u_{3} & =0 .
\end{aligned}
$$

It follows that there are 4 possible steady states: $u_{1}=u_{2}=u_{3}=0$, denoted $E_{000} ; u_{1}, u_{2}>0, u_{3}=0$, denoted $E_{110} ; u_{1}, u_{3}>0, u_{2}=0$, denoted $E_{101}$; and $u_{1}, u_{2}, u_{3}>0$, denoted $E_{111}$. In what follows, we show the existence and stability criteria for all 4 steady states. To this end, it is convenient to note that the Jacobian at any point, $u_{1}, u_{2}, u_{3}$ is

$$
J=\left[\begin{array}{ccc}
-1 & a_{12} & a_{13} \\
-\rho_{2} u_{2} a_{21} & -\rho_{2} u_{2} a_{22}+\rho_{2} f_{2} & -\rho_{2} u_{2} a_{31} \\
-\rho_{3} u_{3} a_{31} & -\rho_{3} u_{3} a_{32} & -\rho_{3} u_{3} a_{33}+\rho_{3} f_{3}
\end{array}\right]
$$

where

$$
\begin{aligned}
& f_{2} \equiv r-a_{21} u_{1}-a_{22} u_{2}-a_{23} u_{3} \\
& f_{3} \equiv r-a_{31} u_{1}-a_{32} u_{2}-a_{33} u_{3}
\end{aligned}
$$

1. $E_{000}=[0,0,0]^{t}$ : It is evident that this steady state always exists. The Jacobian yields no information regarding the stability since it is singular at $E_{000}$. However, we can infer that it is unstable for all $r>0$ by observing that the $u_{2}$-axis is an invariant manifold. The motion along this invariant manifold is given by

$$
\frac{d u_{2}}{d t}=\rho_{2}\left(r-a_{22} u_{2}\right) u_{2},
$$

which drives the system away from $E_{000}$ for all $r>0$.

2. $E_{110}=\left[u_{1}, u_{2}, 0\right]^{t}, u_{1}, u_{2}>0$ : It is easy to check that this steady state exists for all $r>0$, and is given by

$$
u_{3}=0, u_{2}=\frac{r}{\alpha_{22}}, u_{1}=a_{12} u_{2},
$$

where $\alpha_{22} \equiv a_{22}+a_{21} a_{12}$, and the Jacobian at $E_{110}$ is

$$
\left[\begin{array}{ccc}
-1 & a_{12} & a_{13} \\
-\rho_{2} u_{2} a_{21} & -\rho_{2} u_{2} a_{22} & -\rho_{2} u_{2} a_{31} \\
0 & 0 & \rho_{3}\left(r-a_{31} u_{1}-a_{32} u_{2}\right)
\end{array}\right] .
$$

It follows that one of the eigenvalues is

$$
\lambda_{1}=\rho_{3}\left(r-a_{31} u_{1}-a_{32} u_{2}\right)=\rho_{3} r\left(1-\frac{\alpha_{32}}{\alpha_{33}}\right),
$$

where $\alpha_{32} \equiv a_{32}+a_{31} a_{12}$. The other two eigenvalues satisfy

$$
\begin{aligned}
\lambda_{2}+\lambda_{3} & =-1-\rho_{2} a_{22} r u_{2}<0, \\
\lambda_{2} \lambda_{3} & =\rho_{2} a_{22} r u_{2}+\rho_{2} u_{2} a_{21} a_{12}>0,
\end{aligned}
$$

so that the real parts of $\lambda_{2}, \lambda_{3}$ are negative. Hence, $E_{110}$ is stable if and only if $\beta_{32} \equiv \alpha_{32} / \alpha_{22}>1$.

3. $E_{101}=\left[u_{1}, 0, u_{3}\right]^{t}, u_{1}, u_{3}>0$ : The calculations are analogous to those for $E_{101}$.

4. $E_{111}=\left[u_{1}, u_{2}, u_{3}\right]^{t}, u_{1}, u_{2}, u_{3}>0$ : This steady state satisfies the equations

$$
\begin{aligned}
u_{1} & =a_{12} u_{2}+a_{13} u_{3} \\
r & =\alpha_{22} u_{2}+\alpha_{23} u_{3} \\
r & =\alpha_{32} u_{2}+\alpha_{33} u_{3} .
\end{aligned}
$$


It follows that $E_{111}$ exists for all $r>0$ if and only if $[1,1]^{t}$ lies between $\left[\alpha_{22}, \alpha_{32}\right]^{t}$ and $\left[\alpha_{23}, \alpha_{33}\right]^{t}$, i.e., $\beta_{23} \equiv \alpha_{23} / \alpha_{33}, \beta_{32} \equiv \alpha_{32} / \alpha_{22}$ satisfy

$$
\beta_{23}, \beta_{32}<1 \text { or } \beta_{23}, \beta_{32}>1 \text {. }
$$

In both cases, the steady state is given by

$$
\begin{aligned}
& u_{2}=\frac{1}{\alpha_{22}} \frac{1-\beta_{23}}{1-\beta_{23} \beta_{32}} r, \\
& u_{3}=\frac{1}{\alpha_{33}} \frac{1-\beta_{32}}{1-\beta_{23} \beta_{32}} r, \\
& u_{1}=a_{12} u_{2}+a_{13} u_{3} .
\end{aligned}
$$

Evidently, $u_{1}, u_{2}, u_{3}$ increase linearly with $r$.

It turns out that $E_{111}$ is unstable if $\beta_{23}, \beta_{32}>1$, and stable for sufficiently small $r>0$ if $\beta_{23}, \beta_{32}>1$. To see this, observe that the Jacobian at $E_{111}$ is

$$
\left[\begin{array}{ccc}
-1 & a_{12} & a_{13} \\
-\rho_{2} u_{2} a_{21} & -\rho_{2} u_{2} a_{22} & -\rho_{2} u_{2} a_{31} \\
-\rho_{3} u_{3} a_{31} & -\rho_{3} u_{3} a_{32} & -\rho_{3} u_{3} a_{33}
\end{array}\right]
$$

so that

$$
\begin{aligned}
\operatorname{tr} J= & -1-\rho_{2} a_{22} u_{2}-\rho_{3} a_{33} u_{3}, \\
\operatorname{det} J= & -\rho_{2} \rho_{3} u_{2} u_{3} \alpha_{22} \alpha_{23}\left(1-\beta_{23} \beta_{32}\right), \\
\Sigma J= & \rho_{2} \rho_{3} u_{2} u_{3}\left(a_{22} a_{33}-a_{23} a_{32}\right)+\rho_{2} u_{2} \alpha_{22} \\
& \quad+\rho_{3} u_{3} \alpha_{33} .
\end{aligned}
$$

If $\beta_{23}, \beta_{32}>1$, then $\operatorname{det} J>0$, and $E_{111}$ is unstable. Hence, $E_{111}$ is stable only if $\beta_{23}, \beta_{32}<1$. We are particularly interested in the case when $a_{22} a_{33}-a_{23} a_{32}>0$, since this is necessary for Turing instability (see below). Under these conditions, $E_{111}$ is stable for all sufficiently small $r>0$, since $\operatorname{tr} J<0$, det $J<0$ for all $r>0$, and $(\operatorname{tr} J)(\Sigma J)-\operatorname{det} J<0$ for all sufficiently small $r>0$ (Figure 10).

\section{B Turing instability of homogeneous steady states}

\section{B.1 Conditions for Turing instability}

A homogeneous steady state, $u=\left[u_{1}(r), u_{2}(r), u_{3}(r)\right]^{t}$, is Turing unstable if it is stable in the absence of diffusion and unstable in the presence of diffusion. Now the stability of the homogeneous steady state, $u$, in the presence of diffusion is determined by the linearized equation

$$
\frac{\partial v}{\partial t}=D \frac{\partial v}{\partial x^{2}}+J(u) v
$$

where $v$ denotes the deviation from $u, J(u)$ denotes the Jacobian at $u$, and $D=\operatorname{diag}\left(d_{1}, d_{2}, d_{3}\right)$. Let $\mu_{k}$ and $\phi_{k}(x)$ denote the eigenvalues and eigenfunctions of the differential operator, $\frac{d^{2}}{d x^{2}}$, with Neumann boundary conditions, i.e.,

$$
\mu_{k}=-(k \pi)^{2}, \phi_{k}(x)=\cos (k \pi x), k=0,1,2, \ldots
$$

If the steady state is perturbed along any eigenfunction, $\phi_{k}(x)$, the subsequent evolution of the perturbation is of the form $v(t, x)=s_{k}(t) \phi_{k}(x)$ where $s_{k}(t) \in \mathbb{R}^{3}$. Substituting in (14) yields

$$
\frac{d s_{k}}{d t}=C_{k} s_{k}, C_{k}(u) \equiv J(u)-(k \pi)^{2} D .
$$

It follows that the homogeneous steady state is stable if and only if the eigenvalues of $C_{k}(u)$ have negative real parts for all $k \geq 0$. It is Turing unstable if and only if 


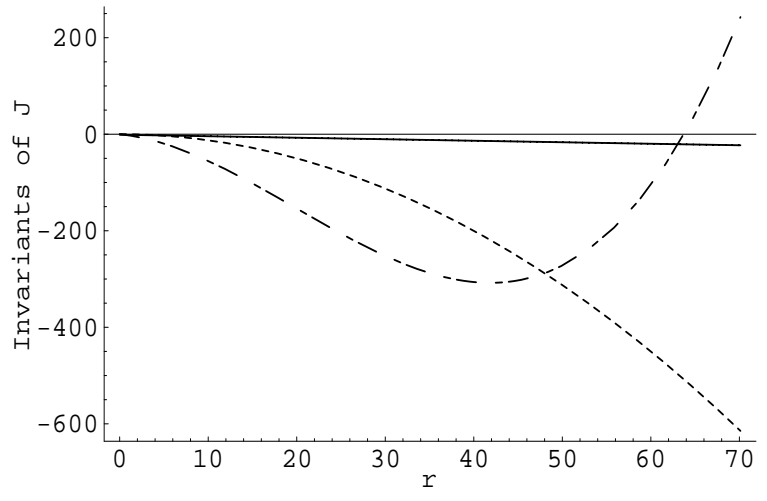

(a)

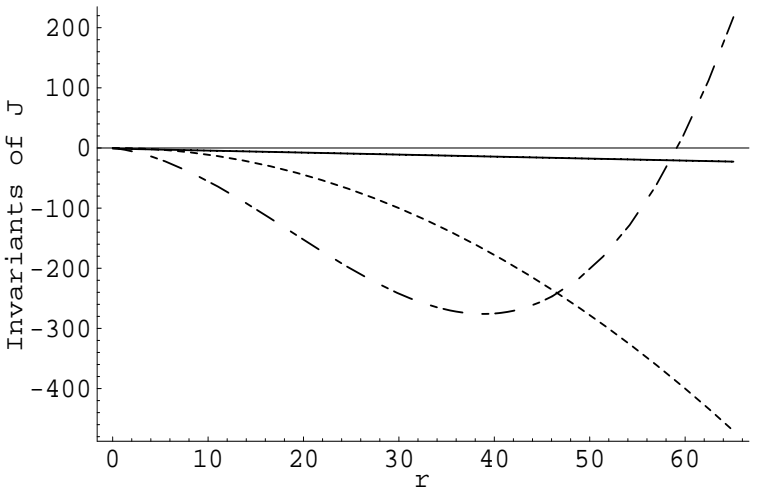

(b)

Figure 10: Variation of $\operatorname{tr} J(-), \operatorname{det} J(--)$, and $(\operatorname{tr} J)(\Sigma J)-\operatorname{det} J(--)$ as a function of $r$. (a) Parameter values chosen such that $U_{2}$ prevails over $U_{3}$ in the absence of $U_{1}$. (b) Parameter values chosen such that $U_{3}$ prevails over $U_{2}$ in the absence of $U_{1}$.

1. The eigenvalues of $C_{0}(u)=J(u)$ have negative real parts (which ensures that the homogeneous steady state is stable in the absence of diffusion).

2. The eigenvalues of $C_{k}(u)$ have positive real part for some $k>0$ (which ensures that the homogeneous steady state is unstable in the presence of diffusion).

The Turing bifurcation occurs at the critical value, $r=r_{0}>0$, such that exactly one of the eigenvalues of $C_{k}(u)$ becomes zero, i.e.,

$$
\operatorname{det} C_{k}(u)=0, \frac{d}{d k^{2}} \operatorname{det} C_{k}(u)=0
$$

for some $k>0$ (Figure 11).

\section{B.2 Turing instability of the homogeneous steady states}

It follows from the definition of $C_{k}$ that 28

$$
\begin{aligned}
& \operatorname{det} C_{k}(u)=\triangle_{123}-\left(d_{1} \triangle_{23}+d_{2} \triangle_{13}+d_{3} \triangle_{13}\right) k^{2} \\
&+\left(d_{1} d_{2} \triangle_{3}+d_{2} d_{3} \triangle_{1}+d_{1} d_{3} \triangle_{2}\right) k^{4} \\
&-\left(d_{1} d_{2} d_{3}\right) k^{6}
\end{aligned}
$$

where $\triangle_{i}, \triangle_{i j}, i \neq j, \triangle_{123}$ are the determinants of the 1-, 2-, and 3-dimensional subsystems of $J$, i.e., $\triangle_{i}=J_{i i}(u)$

$$
\triangle_{i j}=\operatorname{det}\left[\begin{array}{cc}
J_{i i} & J_{i j} \\
J_{j i} & J_{j j}
\end{array}\right], i \neq j
$$

and $\triangle_{123}=\operatorname{det} J(u)$. These results are sufficient for investigating the possibility that the homogeneous steady states undergo a Turing bifurcation.

We consider each of the homogeneous steady states.

1. $E_{000}=[0,0,0]^{t}$ : Since $E_{000}$ is unstable even in the absence of diffusion, it cannot entertain Turing instability. 


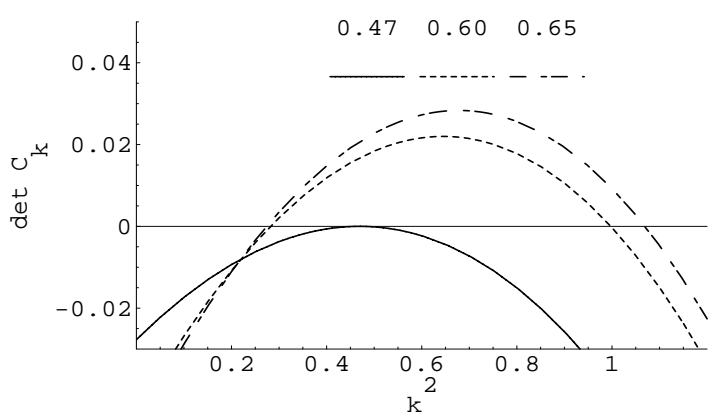

(a)

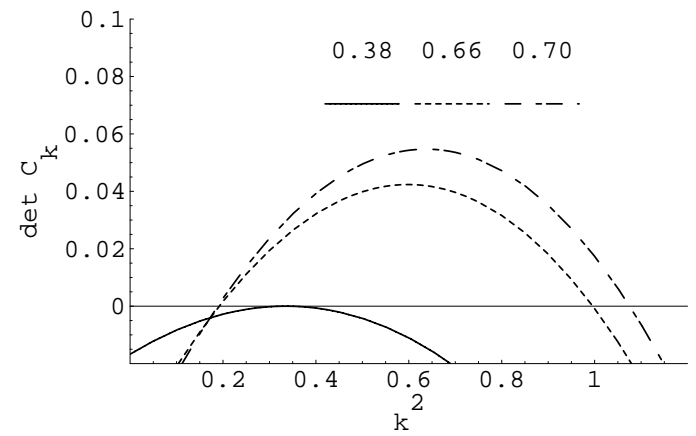

(b)

Figure 11: Variation of $\operatorname{det} C_{k}$ as a function of $k^{2}$ at various values of $r$. (a) Parameter values chosen such that $U_{2}$ prevails over $U_{3}$ in the absence of $U_{1}$. (b) Parameter values chosen such that $U_{3}$ prevails over $U_{2}$ in the absence of $U_{1}$.

2. $E_{110}=\left[u_{1}, u_{2}, 0\right]^{t}$ : This steady state is stable in the absence of diffusion provided $\beta_{32}>1$. Thus, it can undergo a Turing instability if and only if there is an $r>0$ such that (15) has a positive root. It turns out that such a root does not exist. To see this, observe that since $E_{110}$ is stable in the absence of diffusion, $\triangle_{123}=\operatorname{det} J<0$. Inspection of the Jacobian (12) shows that the $\triangle_{i}$ 's are always negative, and the $\triangle_{i j}$ 's are always positive. Hence, all the coefficients of the polynomial (15) are negative for all $r>0$. Such a polynomial cannot have positive roots $(k>0)$. We conclude that $E_{110}$ cannot undergo a Turing instability.

3. $E_{101}=\left[u_{1}, 0, u_{3}\right]^{t}$ : This steady state is stable in the absence of diffusion provided $\beta_{23}<1$. An argument analogous to that for $E_{110}$ shows that it cannot undergo a Turing instability.

4. $E_{111}=\left[u_{1}, u_{2}, u_{3}\right]^{t}$ : This steady state is stable in the absence of diffusion provided $\beta_{23}, \beta_{32}<1$ and $r$ is sufficiently small. Under these conditions, $\triangle_{123}<0$. Inspection of the Jacobian (13) shows that no matter what the value of $r$, all the $\triangle_{i}$ 's are negative, and $\triangle_{12}, \triangle_{13}$ are positive. Hence, $E_{111}$ can undergo a Turing instability only if $\triangle_{23}<0$. This condition is not only necessary, but also sufficient. For, if $d_{2}$ and $d_{3}$ are sufficiently small compared to $d_{1}$, the coefficient of $k^{2}$ is approximately $d_{1} \triangle_{23}<0$, and the polynomial (15) has a positive root.

The non-homogeneous steady state that bifurcates from $E_{111}$ is such that $U_{2}$ and $U_{3}$ are out-of-phase. To see this, it suffices to observe that to a first degree of approximation, the non-homogeneous steady state near the Turing bifurcation point has the form $v_{0} \cos \left(k_{0} x\right)$, where $k_{0}$ be the critical wavenumber, and $v_{0}$ is the eigenvector corresponding to the zero eigenvalue of $C_{k_{0}}$, i.e., $C_{k_{0}} v_{0}=0$. The null space of the rank-2 matrix, $C_{k_{0}}$, is spanned by the vector, $\left[c_{1}, c_{2}, 1\right]$, where

$$
c_{2} \equiv-\frac{C_{k_{0}, 22}-C_{k_{0}, 12} C_{k_{0}, 21} / C_{k_{0}, 11}}{C_{k_{0}, 23}-C_{k_{0}, 21} C_{k_{0}, 13} / C_{k_{0}, 11}}
$$

Substitution of the coefficients of $C_{k_{0}}$ shows that $c_{2}$ is always negative, which implies that $U_{2}$ and $U_{3}$ are out-of-phase. 